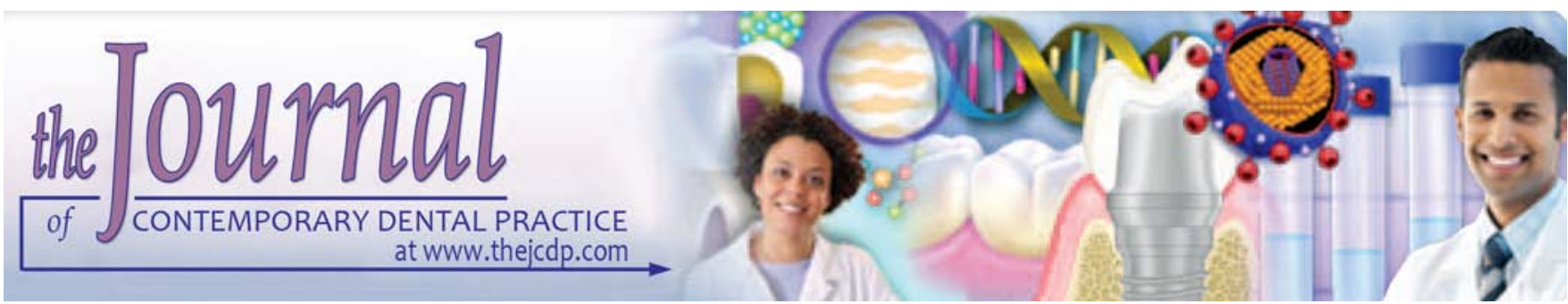

\title{
A Rare Case of Mucoepidermoid Carcinoma ex Pleomorphic Adenoma arising in Minor Salivary Gland: Histopathological and Immunohistochemical Analysis
}

${ }^{1}$ Felipe Perozzo Daltoe, ${ }^{2}$ Liliane Janete Grando, ${ }^{3}$ Maria Inês Meurer, ${ }^{4}$ Elena Riet Correa Rivero, ${ }^{5}$ Filipe Modolo

\begin{abstract}
Mucoepidermoid carcinoma ex pleomorphic adenoma (MCXPA) is a rare salivary gland tumor predominantly found in major salivary glands. A case of MCxPA involving the soft tissue and bone of the retromolar region of a 26 -year-old man is presented. The histopathological features revealed a neoplasm with predominance of pleomorphic adenoma (PA) elements, and presence of mucoepidermoid carcinoma malignant epithelial cells in several areas. Histochemical and immunohistochemical studies were positive for periodic acid Schiff, alcian blue, cytokeratins $7,13,14$, and 19, Bcl-2, c-erbB-2, FGF-2 and maspin in the malignant areas. The patient underwent a partial resection of the left side of the mandible with neck dissection and MCXPA diagnosis was confirmed.
\end{abstract}

Keywords: Ex pleomorphic adenoma, Mucoepidermoid carcinoma, Salivary gland, Immunohistochemistry.

How to cite this article: Daltoe FP, Grando LJ, Meurer MI, Rivero ERC, Modolo F. A Rare Case of Mucoepidermoid Carcinoma ex Pleomorphic Adenoma arising in Minor Salivary Gland: Histopathological and Immunohistochemical Analysis. J Contemp Dent Pract 2015;16(7):603-606.

Source of support: Nil

Conflict of interest: None

\section{INTRODUCTION}

Carcinoma ex pleomorphic adenoma (CxPA) is a pleomorphic adenoma (PA) in which a second malignant neoplasm develops. ${ }^{1}$ In most cases $(75 \%)$, epithelial

\footnotetext{
${ }^{1-5}$ Department of Pathology, Health Sciences Center, Federal University of Santa Catarina, Santa Catarina, Brazil

Corresponding Author: Felipe Perozzo Daltoe, PhD Student Department of Pathology, Health Sciences Center, Federal University of Santa Catarina, University Campus, Trindade CEP: 88040-900, Florianopolis, SC, Brazil, Phone: +55 (48) 3721-3483, e-mail: felipedaltoe@usp.br
}

cells suffer malignant transformation, but in others the carcinoma may show dual epithelial-myoepithelial differentiation (19\%) or pure myoepithelial differentiation $(6 \%)^{2}$

Carcinoma ex pleomorphic adenoma comprises only $3.6 \%$ of all salivary gland tumors, develops predominantly in major salivary glands and appears usually in the 6th or 7th decades of life. ${ }^{3}$ Furthermore, it is very aggressive, commonly metastasizes and usually presents a high mortality rate. ${ }^{4}$ Brandwein et al (1996) classified CxPAs according to the invasion extension beyond the previous PA capsule as intracapsular (without invasion), minimally invasive ( $\leq 1.5 \mathrm{~mm}$ of invasion) and frankly invasive ( $>1.5 \mathrm{~mm}$ of invasion). ${ }^{5}$

Although many subtypes of salivary gland carcinomas have been reported in association with PA, mucoepidermoid carcinoma (MEC) arising from a PA is rare. In this study, a case of mucoepidermoid carcinoma ex pleomorphic adenoma (MCxPA) arising in a minor salivary gland and its clinicopathological and immunohistochemical features is described.

\section{CASE REPORT}

A 26-year-old male jail prisoner was referred to the Stomatology Service of the University Hospital of at the Federal University of Santa Catarina for evaluation of a 6-month history symptomatic mass involving the left side of the mandible. Physical examination revealed a painful, bleeding and exophytic tumoral mass with rubbery consistency and undefined borders. The lesion involved the lingual part of the left posterior alveolar ridge and the retromolar region and measured approximately $5 \times 3 \mathrm{~cm}$ (Fig. 1A). Lymph node swelling was found in the cervical and submandibular ipsilateral zones upon preoperative examination. Radiography and computerized tomography (CT) 
revealed jawbone destruction in adjacent areas of the third molar root and confirmed lymph node involvement (Fig. 1B). Except for the tumor, the patient's medical history was unremarkable.

An incisional biopsy was performed on the superficial soft tissue and in the intraosseous region (followed third molar extraction). A neoplasm of biphasic appearance with a predominance of benign areas of PA and complementary MEC areas was revealed. Pleomorphic adenoma regions were typically made up of sheets of epithelial cells and duct-like structures, with luminal epithelial and myoepithelial cells in a chondromyxoid background (Fig. 1C); in several areas, malignant epithelial nests and islands of cells were observed.

These malignant epithelial cells showed squamous phenotype predominance, although intermediate, mucous-producing and clear cells were also observed. The intracystic component corresponded to less than $25 \%$ of the malignant part; nuclear pleomorphism, bone invasion, and some necrotic areas were also found. Because malignant cells infiltrated over $1.5 \mathrm{~mm}$ into the previous PA capsules, the lesion was classified as a frankly invasive carcinoma due capsule infiltration $>1.5 \mathrm{~mm} .^{5}$ The tumor could be classified as Grade II by AFIP's criteria (Armed Forces Institute of Pathology) ${ }^{7}$ or Grade III according to Brandwein et al (2001). ${ }^{8}$

Histochemical reactions revealed the sialomucin content of mucous-producing cells by alcian blue staining and the presence of clear cells by diastase-sensitive periodic acid-schiff positivity (Fig. 1D).

Immunohistochemical study revealed positivity to cytokeratins (CKs) 7, 13 (Fig. 1E) and 19 in malignant epithelial cells. Cytokeratin 14 (Ck14) was expressed in both benign epithelial and myoepithelial PA cells as well as in squamous and intermediate MEC cells. Luminal malignant epithelial cells (MECs) showed a strong FGF2 staining for c-erbB-2 and weak and focal staining for Bcl-2. In addition, malignant cells showed a strong FGF-2 expression, which was even more intense in the epithelial cells from the peripheral tumor borders. Benign areas of the tumor showed a strong maspin expression (Fig. 1F). There were no immunohistochemical expressions of vimentin, p53, Ki-67 and alpha smooth muscle actin $(\alpha-S M A)$ in malignant epithelial cells. The myoepithelial component of PA and stroma expressed vimentin. The final histopathological diagnosis was MCxPA and the clinical TNM-classification was T4N2M0.

The patient underwent a partial resection of the left jaw and a modified neck dissection (levels I to V lymph nodes ipsilateral) and the entire specimen was submitted to histopathological examination. The diagnosis was confirmed and there was no metastasis evidence in the lymph nodes. The patient follow-up was not possible because he escaped from jail before mandible reconstruction.

\section{DISCUSSION}

Although PA is a benign and well-encapsulated tumor, there are reports of recurrence malignant transformation. ${ }^{3,5,13}$ Carcinoma ex pleomorphic adenoma arises most frequently in parotid and submandibular glands, but can occur in the palate as well. Other sites are rare. 3,9

This case report present a MCXPA developing uncommon location (alveolar ridge and retromolar trigone) and in a younger than-average patient. Even though many subtypes of salivary gland carcinomas have been reported in association with PA, the existence of MEC arising in PA has rarely been reported. $3,4,6$

Histologically, the malignant compound of this MCxPA case can be classified as Grade II by AFIP criteria (Armed Forces Institute of Pathology) or Grade III according to Brandwein et al (2001). ${ }^{7,8}$ This difference in graduation can be explained as a tendency to downgraduate MEC by AFIP's criteria. ${ }^{8}$

Immunostaining of CK7, CK14 and CK19 helps to differentiate primary salivary gland neoplasms from metastatic tumors and squamous cell carcinoma. ${ }^{10}$ Furthermore, CK13 staining is very usefull for differentiate diagnosis, among salivary gland tumors, once only canalicular adenoma and mucoepidermoid carcinoma express to this protein. ${ }^{11}$ Immunohistochemical staining for maspin helps to evaluate the clinical behavior of CxPA once according to Martins et al (2005), the less aggressive salivary gland tumors are the higher immunostaining for maspin they present. ${ }^{12}$ In this case, the PA component showed a moderate presence of maspin while the malignant component showed weak or absence of immunostaining.

According to Freitas et al (2005), c-erbB-2 reactivity is strongly associated with atypical luminal cells. ${ }^{13}$ In agreement with this, our findings showed c-erB-2 expression in the malignant counterpart luminal cells. Additionally, this case showed a weak and focal Bcl-2 expression in malignant areas, similarly to the one reported in two CxPA cases in their early stages which were studied by the same author. ${ }^{13}$

No staining for p53 was observed in our case. The expression of this protein has been considered uncommon in salivary gland tumors and mostly associated with aggressive clinical behavior (expressed by large, more invasive and higher grade tumors). ${ }^{14}$

Although Ki-67 is considered a useful marker to distinguish PA from CxPA, its expression was not observed in the present case. ${ }^{13}$ On the other hand, a strong FGF-2 
expression was observed in malignant epithelial cells, especially by those from the front invasion islets. As also suggested by Furuse et al (2010), this finding might the importance of this protein in tumor growth, invasion and metastasis. ${ }^{15}$

It was very important to know that MEC was the malignant component of this tumor, once CxPA treatment and prognosis are based on the type of the malignant counterpart. Patients with MEC are usually treated with surgical resection and have a favorable outcome, with a low metastasis potential; whereas invasive CxPA, as a group, is extremely aggressive with a range of 23 to $50 \%$ of the patients developing one or more recurrences and a metastatic rate up to $70 \%$ (mainly when tumor capsule penetration exceeds $1.5 \mathrm{~mm}$ ) So, for a MCxPA presenting frankly invasive characteristics, a complete surgical excision followed by lymph node dissections without radiotherapy complement was performed. ${ }^{5}$
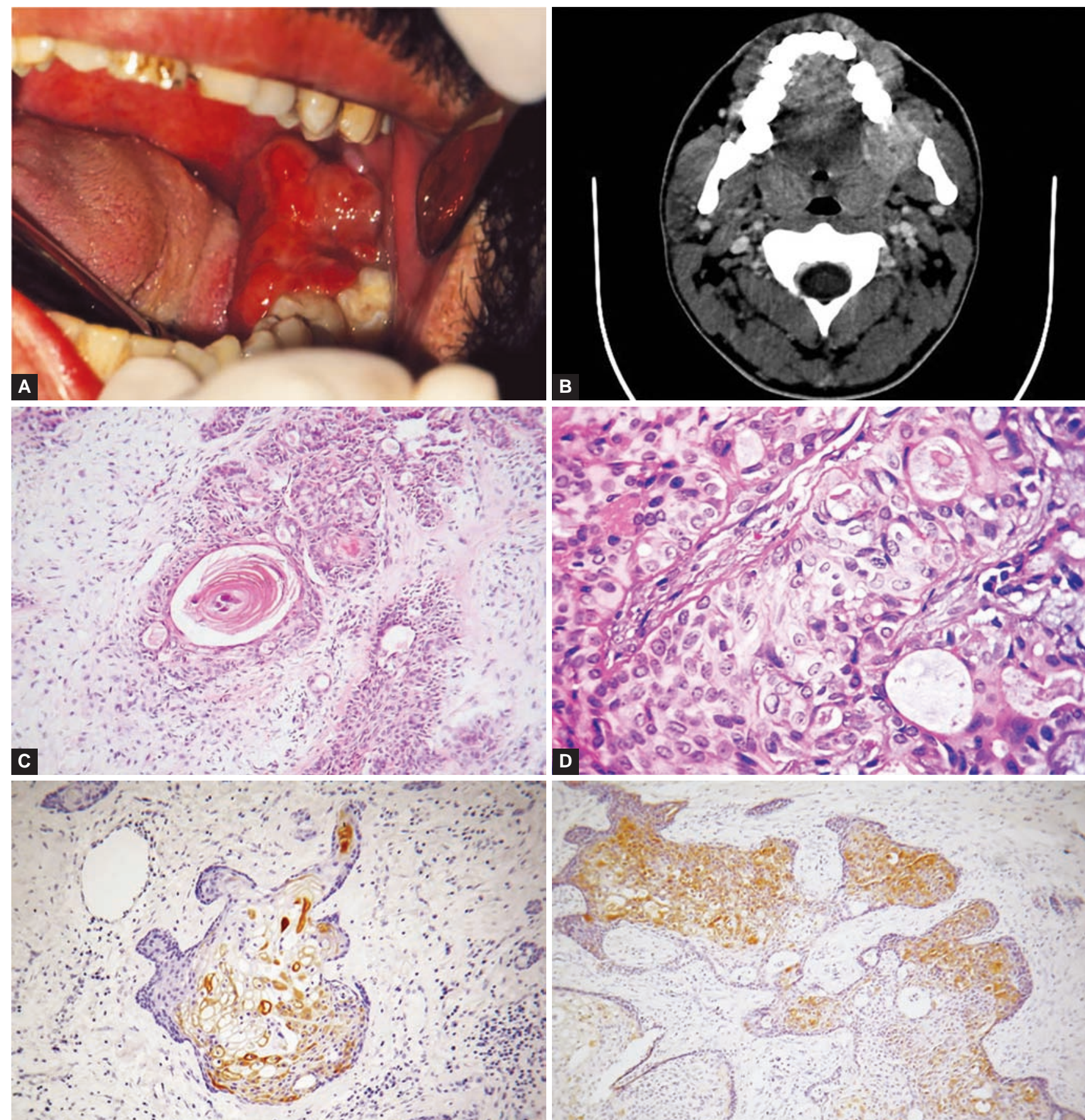

E

E.

Figs $1 \mathrm{~A}$ to $\mathrm{F}$ : Clinical, imaginologic and histopathological aspects of the lesion: (A) Extensive painful exophytic mass in the retromolar region of the left mandible covered by reddish mucosa, $(B)$ computerized tomography in axial view revealing bone destruction on the posterior left mandible, (C) benign counterpart of the tumor showing epithelial duct-like structures and squamous metaplasia surrounded by a myxoid/hyalinised stroma, (D) PAS stain evidencing scattered positive malignant mucous cells, (E) positivity to CK13 in malignant epithelial cells and (F) expression of maspin in benign areas 
New approaches on the molecular genetics field will certainly help us to better understand MCXPA etiopathogenesis and behavior in the future. Recent groups have shown that $70 \%$ of the pleomorphic adenomas are related to specific genetic alterations, and specific chromosomal translocation has been recognized in mucoepidermoid carcinoma. $^{2}$

In conclusion, this case report emphasizes the importance of the correlation among clinical, imaginologic and histopathological aspects in order to reach an appropriate diagnosis. Although on first observation, the predominance of PA elements led us to believe in a benign neoplasm, the aggressive clinical behavior instigated us to perform a histochemical and immunohistochemical study to confirm potential malignancy.

\section{REFERENCES}

1. Gnepp DR. Malignant mixed tumors of the salivary glands: a review. Pathol Annu 1993;28(1):279-328.

2. Cheuk W, Chan JK. Advances in salivary gland pathology. Histopathology 2007;51(1):1-20.

3. Gnepp DR, Brandwein-Gensler MS, El-Naggar AK, Nagao T. World Health Organization Classification of Tumours. Pathology and genetics of head and neck tumours. Lyon: IARC Press; 2005. Chapter 5, Salivary glands; p. 243-244.

4. Araújo VC, Demasi AP, Furuse C, Altemani A, Alves VA, Freitas LL, Araújo NS. Collagen type I may influence the expression of e-cadherin and beta-catenin in carcinoma ex-pleomorphic adenoma. Appl Immunohistochem Mol Morphol 2009;17(4):312-318.

5. Brandwein M, Huvos AG, Dardick I, Thomas MJ, Theise ND. Noninvasive and minimally invasive carcinoma ex mixed tumor: a clinicopathologic and ploidy study of 12 patients with major salivary tumors of low (or no?) malignant potential. Oral Surg Oral Med Oral Pathol Oral Radiol Endod 1996;81(6):655-664.

6. Pitman MB. Mucoepidermoid carcinoma ex pleomorphic adenoma of the parotid gland. Acta Cytol 1995;39(3):604-606.

7. Goode RK, Auclair PL, Ellis GL. Mucoepidermoid carcinoma of the major salivary glands: clinical and histologic analysis of 234 cases with evaluation of grading criteria. Cancer 1998;82(7):1217-1224.

8. Brandwein MS, Ivanov K, Wallace DI, Hille JJ, Wang B, Fahmy A, Bodian C, Urken ML, Gnepp DR, Huvos A, et al. Mucoepidermoid Carcinoma A clinicopathologic study of 80 patients with special reference to histological grading. Am J Surg Pathol 2001;29(7):835-845.

9. Livolsi VA, Perzin K. Malignant mixed tumors arising in salivary glands. Cancer 1977;39(5):2209-2230.

10. Meer S, Altini M. CK7+/CK20 immunoexpression profile is typical of salivary gland neoplasia. Histopathology 2007;51 (1):26-32.

11. de Araújo VC, de Sousa SO, Carvalho YR, de Araújo NS. Application of immunohistochemistry to the diagnosis of salivary gland tumors. Appl Immunohistochem Mol Morphol 2000;8(3):195-201.

12. Martins MT, Altemani A, Freitas L, Araújo VC. Maspin expression in carcinoma ex pleomorphic adenoma. J Clin Pathol 2005;58(12):1311-1314.

13. Freitas LL, Araújo VC, Martins MT, Chone C, Crespo A, Altemani A. Biomarker analysis in carcinoma ex pleomorphic adenoma at an early phase of carcinomatous transformation. Int J Surg Pathol 2005;13(4):337-342.

14. Lewis JE, Olsen KD, Sebo TJ. Carcinoma ex pleomorphic adenoma: pathologic analysis of 73 Cases. Human Pathology 2001;32(6):596-604.

15. Furuse C, Miguita L, Rosa AC, Soares AB, Martinez EF, Altemani A, de Araújo VC. Study of growth factors and receptors in carcinoma ex pleomorphic adenoma. J Oral Pathol Med 2010;39(7):540-547. 